\title{
PHENOLICS, DEPSIDES AND TRITERPENES FROM THE CHILEAN LICHEN PSEUDOCYPHELLARIA NUDATA (ZAHLBR.) D.J. GALLOWAY
}

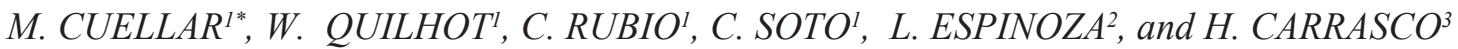

\begin{abstract}
${ }^{1}$ Departamento de Ciencias Químicas y Recursos Naturales, Facultad de Farmacia, Universidad de Valparaíso, Av. Gran Bretaña No 1093, Valparaíso, Chile. ${ }^{2}$ Departamento de Química, Universidad Técnica Federico Santa María, Av. España No 1680, Valparaíso, Chile.

${ }^{3}$ Departamento de Ciencias Químicas, Universidad Andrés Bello, Campus Viña del Mar, Los Fresnos № 52, Viña del Mar, Chile. (Received: January 23, 2008 - Accepted: April 28, 2008)
\end{abstract}

\begin{abstract}
The lichen Pseudocyphellaria nudata is a species endemic to southern South America. From the lichen tallus, methyl orsellinate, 2-methoxy-3,6-dimethyl4-hydroxybenzaldehyde, methyl-evernate, tenuiorin, hopan-6ß,22-diol and hopan-6 $\alpha, 7 \beta, 22$-triol were isolated and identified as the main lichen constituents. This is the first report of the occurrence of 2-methoxy-3,6-dimethyl-4-hydroxybenzaldehyde in lichens.
\end{abstract}

Keywords: Pseudocyphellaria nudata, lichen, monophenols, depsides, triterpenes.

\section{INTRODUCTION}

In the cool temperate rainforests of southern South America, lichen species belonging to genus Pseudocyphellaria are common'. The genus Pseudocyphellaria is represented in Chile by 54 species occurring from latitude $23^{\circ} \mathrm{S}$ to $56^{\circ} \mathrm{S}$. The mains constituents of the species have been identified by thin layer chromatography ${ }^{1,2}$. Pseudocyphellaria nudata (Zahlbr.) D.J. Galloway (Lobariaceae, lichenized Ascomycota) is endemic to southern South America. It is a robust, coriaceous lichen, with the cyanobacteria Nostoc as photobiont. The genus Pseudocyphellaria is characterized by a wide chemical diversity, including substances from the major secondary metabolism pathways. The acetate polymalonate pathway produces depsides, depsidones, and usnic acid; from the shikimic acid pathway, terphenylquinones and principally pulvinic acid derivatives are produced while the mevalonic acid pathway led to sterols and terpenoids ${ }^{3}$. The occurrence of triterpenoids, especially the hopane derivatives, produced also by cyanobacteria and other organisms $\mathrm{s}^{4}$, have attracted considerable interest in the organic geochemistry as indicators of sources of sedimentary organic matter and biomarkers of mature petroleumcontaining layers ${ }^{5,6,7}$. According to Galloway ${ }^{1}$, "the identity of the compounds appearing on thin layer chromatograms remain to be definitively characterized, and that provides an exciting opportunity for future work".

The aim of this work was the identification of the main constituents of a Chilean collection of $P$. nudata by spectroscopic means.

\section{EXPERIMENTAL}

\section{General experimental procedures}

The ${ }^{1} \mathrm{H}$ and ${ }^{13} \mathrm{C}$ NMR spectra were recorded in a Bruker AVANCE spectrometer model 400 operating at $400 \mathrm{MHz}$ for ${ }^{1} \mathrm{H}$ and $100 \mathrm{MHz}$ for ${ }^{13} \mathrm{C}$, using $\mathrm{CDCl}_{3}$ and DMSO- $\mathrm{d}_{6}$ as solvents. Chemical shifts are reported in $\delta \mathrm{ppm}$ and coupling constants $(J)$ are given in Hz. Melting points were determined on a Stuart-Scientific SMP3 apparatus. Optical rotation were measured with a sodium lamp ( $\lambda=589 \mathrm{~nm}$, D line) on a Perkin Elmer 241 digital polarimeter equipped with $1 \mathrm{dm}$ cells. Column chromatography (CC) used silica gel Merck $60 \mathrm{G}(0,032-0,063 \mathrm{~nm})$, the fractions were monitored by thin-layer chromatography (TLC) used chromatoplates silica gel Merck $60 \mathrm{~F}_{254}$. The spots were visualized under UV light $(254 / 365 \mathrm{~nm})$ and developed using $\mathrm{H}_{2} \mathrm{SO}_{4}$ spray reagent ${ }^{1}$.

\section{Plant material.}

Pseudocyphellaria nudata (Zahlbr.) D.J. Galloway (118 g) was collected from bark of Nothofagus spp. at 1000-1100 m altitude in Conguillio National Park $\left(39^{\circ}, 39^{`} \mathrm{~S} ; 7^{\circ}, 43^{\prime} \mathrm{W}\right)$, Chile, in November 2005. The species was determined by W. Quilhot. Voucher specimens are deposited in the Lichen Herbarium (UV), Facultad de Farmacia, Universidad de Valparaíso

Extraction and isolation: The air dried lichen sample was ground, and extracted successively with chloroform and acetone $(2 \mathrm{~L})$, each time $72 \mathrm{~h}$ at room temperature. The solvents were removed under reduced pressure to give a chloroform $(6.08 \mathrm{~g})$ and acetone $(4.50 \mathrm{~g})$ extracts.

The chloroform extract was chromatographed on silica gel using a mixture of dichloromethane and ethyl-acetate with increasing polarity (49:1 and 1:49), collecting $8 \mathrm{ml}$ in each fraction (260 fractions). The fractions were combined based on TLC and ${ }^{1} \mathrm{H}-\mathrm{NMR}$ monitoring. Fractions 5-8 were combined and reduced to dryness. Recrystallisation in $\mathrm{Et}_{2} \mathrm{O}: \mathrm{MeOH}(1: 1)$ afforded an impure precipitate which was removed from the solution, and further concentration led to the isolation of a pure precipitated compound, identified as methyl evernate (1) $(5.8 \mathrm{mg})$, colorless crystalline powde, $\mathrm{mp} 137-138^{\circ} \mathrm{C} .{ }^{1} \mathrm{H}-\mathrm{NMR}\left(\mathrm{CDCl}_{3}\right)$ : $11.57\left(1 \mathrm{H}, \mathrm{s}, \mathrm{OH}-2^{\prime}\right) ; 11.35(1 \mathrm{H}, \mathrm{s}, \mathrm{OH}-2) ; 6.71\left(1 \mathrm{H}, \mathrm{d}, \mathrm{J}=2.3 \mathrm{~Hz}, \mathrm{H}-3^{3}\right)$; $6.60\left(1 \mathrm{H}, \mathrm{d}, \mathrm{J}=2.3 \mathrm{~Hz}, \mathrm{H}-5^{\prime}\right) ; 6.38(1 \mathrm{H}, \mathrm{d}, \mathrm{J}=2.7 \mathrm{~Hz}, \mathrm{H}-5) ; 6.37(1 \mathrm{H}, \mathrm{d}, \mathrm{J}$ $=2.7 \mathrm{~Hz}, \mathrm{H}-3) ; 3.98\left(3 \mathrm{H}, \mathrm{s}, \mathrm{CO}_{2} \mathrm{Me}\right) ; 3.83(3 \mathrm{H}, \mathrm{s}, \mathrm{OMe}) ; 2.62(3 \mathrm{H}, \mathrm{s}, \mathrm{H}-8)$; $2.58\left(3 \mathrm{H}, \mathrm{s}, \mathrm{H}-8^{\prime}\right) .{ }^{13} \mathrm{C}-\mathrm{NMR}\left(\mathrm{CDCl}_{3}\right)$; 171.7 (C-7'); 169.7 (C-7); 166.5 (C-4'); 164.8 (C-4); 164.4 (C-2); 153.9 (C-2'); 143.4 (C-6, C-6'); 116.6 (C-5'); 111.9 (C-5); 110.4 (C-1'); 108.8 (C-3'); 104.3 (C-1); 98.9 (C-3); 55.4 (OMe); 52.3 $\left(\mathrm{CO}_{2} \mathrm{Me}\right) ; 24.6(\mathrm{C}-8) ; 24.2\left(\mathrm{C}-8^{\prime}\right)$ in accordance with literature ${ }^{8}$.

Fractions 16-38 afforded tenuiorin $(2)(1.61 \mathrm{~g})$, white crystalline powder, mp 180-182 ${ }^{\circ} \mathrm{C} .{ }^{1} \mathbf{H}-\mathbf{N M R}\left(\mathrm{CDCl}_{3}\right): 11.61(1 \mathrm{H}, \mathrm{s}, \mathrm{OH}-2$ '”); $11.34(1 \mathrm{H}, \mathrm{s}, \mathrm{OH}-$ 2); $11.15\left(1 \mathrm{H}, \mathrm{s}, \mathrm{OH}-2^{\prime}\right) ; 6.78\left(1 \mathrm{H}, \mathrm{d}, \mathrm{J}=1.5 \mathrm{~Hz}, \mathrm{H}-3^{\prime}\right) ; 6.73(1 \mathrm{H}, \mathrm{d}, \mathrm{J}=1.5$ $\mathrm{Hz}, \mathrm{H}-3$ ' '); $6.69(1 \mathrm{H}, \mathrm{d}, \mathrm{J}=1.5 \mathrm{~Hz}, \mathrm{H}-5$ '); $6.61(1 \mathrm{H}, \mathrm{d}, \mathrm{J}=1.5 \mathrm{~Hz}, \mathrm{H}-5$ ") $) 6.38$ $(2 \mathrm{H}, \mathrm{s}, \mathrm{H}-3+\mathrm{H}-5) ; 3.99$ (3H, s, $\left.\mathrm{CO}_{2} \mathrm{Me}\right) ; 3.84(3 \mathrm{H}, \mathrm{s}, \mathrm{OMe}) ; 2.70\left(3 \mathrm{H}, \mathrm{s}, \mathrm{H}-8^{\prime}\right)$; $2.64(3 \mathrm{H}, \mathrm{s}, \mathrm{H}-8) ; 2.59$ (3H, s, H-8 $\left.{ }^{2 \prime}\right) .{ }^{13} \mathrm{C}-\mathrm{NMR}\left(\mathrm{CDCl}_{3}\right): 171.7$ (C-7'”); 169.6 (C-7)*; 169.3 (C-7')*; 166.6 (C-4')*; 165.2 (C-4'”)*; 164.9 (C-4); 164.4 (C-2); 154.8 (C-2'); 153.6 (C-2'”) $; 143.8$ (C-6'); 143.6 (C-6”'); 143.4 (C-6); 117.2 (C-5'); 116.4 (C-5'); 112.0 (C-5); 110.7 (C-1''); 109.6 (C-1'); 109.2 (C-3'); 108.7 (C-3'); 104.2 (C-1); 98.9 (C-3); 55.4 (OMe); 52.4 (CO $\left.\mathrm{CO}_{2} \mathrm{Me}\right) ; 24.6$ (C-8); 24.5 (C-8'); 24.2 (C-8" ). ${ }^{\dagger}, *, "$ assignment may be interchanged, in accordance with literature ${ }^{8}$

From fractions $42-50$ a residue was obtained which was recrystallized in $\mathrm{Et}_{2} \mathrm{O}: \mathrm{MeOH}(1: 1)$ to afford methyl orsellinate $(3)(16 \mathrm{mg})$, colorless crystalline powder, mp $142-143^{\circ} \mathrm{C} .{ }^{1} \mathrm{H}-\mathrm{NMR}\left(\mathrm{CDCl}_{3}\right): 11.78(1 \mathrm{H}, \mathrm{s}, \mathrm{OH}-2) ; 6.28(1 \mathrm{H}, \mathrm{d}$, $\mathrm{J}=2.1 \mathrm{~Hz}, \mathrm{H}-5) ; 6.23(1 \mathrm{H}, \mathrm{d}, \mathrm{J}=2.1 \mathrm{~Hz}, \mathrm{H}-3) ; 5.63(1 \mathrm{H}, \mathrm{bs}, \mathrm{OH}-4) ; 3.92$ $\left(3 \mathrm{H}, \mathrm{s}, \mathrm{CO}_{2} \mathrm{Me}\right) ; 2.48$ (3H, s, Me-8). ${ }^{13} \mathbf{C}-\mathbf{N M R}\left(\mathrm{CDCl}_{3}\right): 172.1$ (C-7); 165.3 (C-4); 160.3 (C-2); 144.0 (C-6); 111.4 (C-5); 105.6 (C-1); 101.3 (C-3); 51.9 $\left(\mathrm{CO}_{2} \mathrm{Me}\right) ; 24.2(\mathrm{C}-8)$ in accordance with literature ${ }^{8}$.

Fractions 78-92 yielded the compound hopan-7ß,22-diol (4) (13.1 mg), colorless crystalline powder, mp $230-231^{\circ} \mathrm{C},[\alpha]_{\mathrm{D}}{ }^{25}=+26.3^{\circ}\left(\mathrm{c} 0.54 \mathrm{CHCl}_{3}\right)$. ${ }^{1} \mathrm{H}-N M R\left(\mathrm{CDCl}_{3}\right): 3.89(1 \mathrm{H}, \mathrm{dd}, \mathrm{J}=5.0,10.9 \mathrm{~Hz}, \mathrm{H}-7) ; 2.23(1 \mathrm{H}, \mathrm{m}, \mathrm{H}-21)$; 1.21 (3H, s, Me-29); 1.17 (3H, s, Me-30); $1.04(3 \mathrm{H}, \mathrm{s}, \mathrm{Me}-28) ; 0.98$ (3H, s, Me-27); 0.86 (3H, s, Me-23); 0.80 (3H, s, Me-25); 0.79 (3H, s, Me-24); 0.77 $(3 \mathrm{H}, \mathrm{s}, \mathrm{Me}-28) .{ }^{13} \mathrm{C}-\mathbf{N M R}\left(\mathrm{CDCl}_{3}\right): 73.8$ (C-22); 73.5 (C-7); 53.5 (C-17); 53.2 (C-5); 51.1 (C-21); 50.5 (C-9); 50.1 (C-13); 47.7 (C-8); 44.1 (C-18); 43.4 (C14); 41.8 (C-3), 41.4 (C-19); 40.2 (C-1); 38.4 (C-15); 37.4 (C-10); 33.2 (C-23); 33.0 (C-4); 30.8 (C-30); 29.6 (C-6); 28.8 (C-29); 26.4 (C-20); 24.1 (C-12); 22.3 (C-16); 21.5 (C-24); 20.7 (C-11); 18.6 (C-2); 17.7 (C-27); 16.1 (C-28); 15.5 (C-25); 11.2 (C-26) in accordance with literature 8 .

Fractions 148-187 yielded the compound hopan-6 $\alpha, 7 \mathbf{7 3 , 2 2 - t r i o l ~ ( 5 ) ~ ( 1 . 4 6 ~}$ g), colorless crystalline powder, $\mathrm{mp} 226-227^{\circ} \mathrm{C},[\alpha]_{\mathrm{D}}^{25}=+46.9^{\circ}$ (c 0.62 Py). ${ }_{1}$ H-NMR $\left(\mathrm{CDCl}_{3}\right): 3.72(1 \mathrm{H}, \mathrm{dd}, \mathrm{J}=8.6,10.7 \mathrm{~Hz}, \mathrm{H}-6) ; 3.57(1 \mathrm{H}, \mathrm{d}, \mathrm{J}=8.6$ Hz, H-7); 2.23 (1H, m, H-21); 1.20 (3H, s, Me-29); 1.18 (3H, s, Me-30); 1.15 $(3 \mathrm{H}, \mathrm{s}, \mathrm{Me}-23) ; 1.05$ (6H, s, Me-26 + Me-27); 1.00 (3H, s, Me-24); $0.88(3 \mathrm{H}$, s, Me-25); 0.77 (3H, s, Me-28). ${ }^{13}$ C-NMR (CDCl) : 79.4 (C-7); 73.8 (C-22); 73.3 (C-6); 56.8 (C-5); 53.5 (C-17); 51.0 (C-21); 49.9 (C-13); 49.6 (C-9); 47.3 (C-14); 44.0 (C-18); 43.6 (C-19); 43.5 (C-8); 41.4 (C-3); 40.3 (C-1, C-15); 38.8 (C-10); 36.3 (C-23); 33.6 (C-4); 30.8 (C-30); 28.8 (C-29); 26.4 (C-20); 23.9 
(C-12); 22.2 (C-16); 22.0 (C-24); 20.7 (C-11); 18.5 (C-2); 17.7 (C-27); 16.7 (C-25, C-26); 16.1(C-28) in accordance with literature ${ }^{8}$.

The acetone extract was chromatographed on silica gel using a mixture of dichloromethane and ethyl-acetate with increasing polarity (48:2 and 2:48), collecting $8 \mathrm{ml}$ in each fraction ( 230 fractions). Fractions 8 - 14 afforded tenuiorin (3) (248 mg) and the fractions 120-131 yielded compound hopan-6 $\alpha, 7 \boldsymbol{7 3 , 2 2 -}$ triol (5) (284 mg), both compound previously found in the chloroform extract. Fractions 165-183 were combined and reduced to dryness. Recrystallisation in $\mathrm{Et}_{2} \mathrm{O}: \mathrm{MeOH}(1: 1)$ afforded an impure precipitate which was removed from the solution, and further concentration led to isolation of a pure precipitated compound, identified as 2-methoxy-3,6-dimethyl-4-hydroxybenzaldehyde (6) $\left(20 \mathrm{mg}\right.$ ), colorless crystalline powder, $\mathrm{mp} 155-156^{\circ} \mathrm{C}$. ${ }^{1} \mathrm{H}$ - NMR (DMSO$\left.\mathrm{d}_{6}\right): 10.42(1 \mathrm{H}, \mathrm{s}, \mathrm{H}-7) ; 7.06(1 \mathrm{H}, \mathrm{s}, \mathrm{H}-5) ; 3.89(3 \mathrm{H}, \mathrm{s}, \mathrm{OMe}) ; 2.47$ (3H, s, H-9); 2.17 (3H, s, H-8). ${ }^{13}$ C-NMR (DMSO-d ): 186.9 (C-7); 162.6 (C-2); 151.0 (C4); 137.6 (C-6); 114.5 (C-1); 113.2 (C-3); 112.9 (C-5); 21.7 (C-9); 9.7 (C-8) in accordance with literature ${ }^{8}$
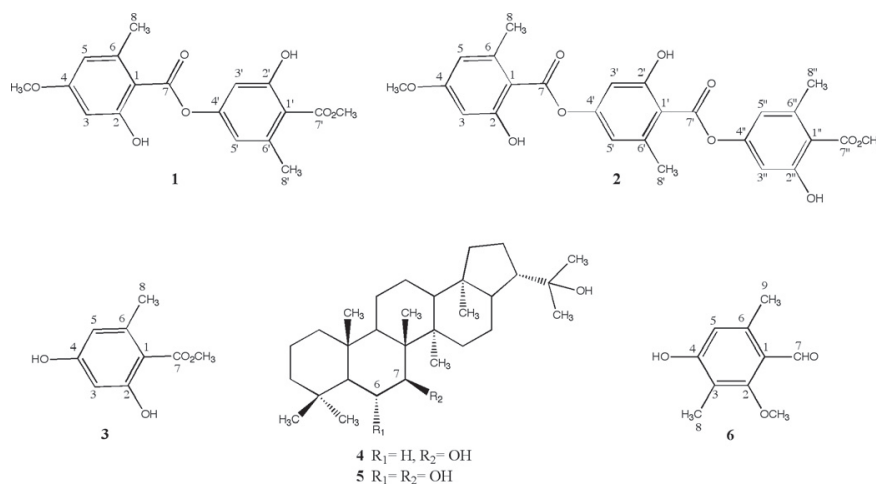

Fig. 1.- Metabolites isolated from P. nudata.

\section{RESULTS AND DISCUSSION}

In the family Lobariaceae the most complex chemistry is found in Pseudocyphellaria; it has the most richly diverse chemistry of any genus, with several compounds synthesized via the shikimic acid pathway ${ }^{8,9,10}$ in addition to contributions from other biosynthetic pathway. The genus is also especially rich in triterpenoids from several different series (hopane, stictane secostictane, lupane and fernene series), exhibiting an evolution in chemical complexity which reflect both geological age and phylogenetic relationships ${ }^{11,12}$.

The compounds isolated from $P$. nudata were previously reported from several species of the genus. Since, with the exception of the triterpene hopane$6 \alpha, 7 \beta, 22$-triol (5), other compounds informed in $P$. nudata by means of TLC, including stictic, constictic acid, cryptostic and constictic acids, methyl gyrophorate $^{1}$, were not detected, probably because the low concentrations of the compounds in the thalli.

Tenuiorin (2) and methyl orsellinate (3) are currently found in species of Pseudocyphellaria and in other lichen genus ${ }^{3}$.

The phenolic 2-methoxy-3,6-dimethyl-4-hydroxybenzaldehyde (6), however, is reported for the first time from a lichen. It was isolated from the fungus Aspergillus silvaticus ${ }^{13}$, where additionally were reported metabolites related to 3-methylorsellinate; also methyl $\beta$-orcinolcarboxylate was isolated from Stereocaulon alpinum ${ }^{14}$

Hopanoids are pentacyclic triterpenes occurring predominantly in bacteria ${ }^{15}$. It has been suggested that the compounds are of great importance as cell membrane stabilizers in bacteria ${ }^{16}$. Hopanoids were discovered in nitrogenfixing bacteria ${ }^{17,18}$, and also occurs in soil bacteria as Bradyrhizobium root nodules in legume plants ${ }^{19}$.

The hopanoid hopan-7ß,22-diol (4) has been isolated from $P$. impressa and $P$ crocata ${ }^{4,9}$, and hopan-6 $\alpha, 7 ß, 22$-triol (5) from P. crocata and Nephroma laevigatum ${ }^{10}$. Hopanoids are very common in lichens with green algae and/or cyanobacteria as primary or secondary photobionts ${ }^{20}$.

\section{ACKNOWLEGMENTS}

This research was supported by the grants DIPUV 21/2005 and DIPUV 22/2005 from Dirección de Investigación, Universidad de Valparaíso. Our thanks to Corporación Nacional Forestal (IX Región) for logistic assistance in the field work.

\section{REFERENCES}

1. D. J. Galloway, Biblioth. Lichenol. 46, 1, (1992).

2. J.W. Bjerke, D.J. Galloway, A. Elvebbak, W. Quilhot, Crypt. Mycol. 24, 59, (2003).

3. C. Culberson, W.L. Culberson, A. Johnson Am. Bryol. Lichenol. Soc., Missouri Botanical Garden, St. Louis, 1977.

4. J.D. Connolly, R.A. Hill Dictionary of Terpenoids Chapman \& Hall, 1991.

5. S. Sakata, J.M Hayes, A.R. Mc Taggart, R.A Evans, K.J. Leckrone, R.K. Togasaki, Geochim. Cosmochim. Acta, 61, 5379, (1997).

6. S. Hanish, D. Ariztegui, W. Püttmann, Org. Geochem. 34, 1223, (2003).

7. J.S. Sinninghe Damsté, I.C. Rijpstra, S. Schouten, J. Fuerst, M.S.M. Jetten , M.. Strous, Org. Geochem. 35, 561, (2004).

8. S. Huneck, I. Yoshimura, Identification of Lichen Substances, SpringerVerlag, Berlin-Heildelberg-New York, 1996.

9. J.A. Garbarino, W. Quilhot, M. Piovano, C. Rubio, Bol. Soc. Chil. Quim. 36, 229, (1991).

10. M. Piovano, M.C. Chamy, J.A. Garbarino. Bol. So. Chil. Quím. 46, 1, (2001).

11. D.J. Galloway, J. Linn. Soc. Bot. 96, 45, (1988).

12. D.J. Galloway, Symbiosis, 11, 327, (1991).

13. N. Kawahara, K. Nozawa, S. Nakajima, S.I. Udagawa, K.I. Kawai, Chem Pharm. Bull. 36, 398, (1988).

14. J. P. Hylands, K. Ingolfsdottir, Phytochemistry, 24, 127, (1985).

15. G. Ourisson, M. Rohmer, K. Poralla, Annu. Rev. Microbiol. 41, 301, (1987).

16. K. Poralla, E. Kannenberg, A. Blume, FEBS Lett. 113, 107, (1980).

17. A.N. Berry, R.A. Moreau, A.D. Jones, Plant Physiol. 95, 111, (1991).

18. C. Vilchezc, P. LLopiz, P. Neunlist, K. Poralla, M. Rohmer, Microbiol. 140, 2749, (1994).

19. E.L. Kannenberg, M. Perzl, Th. Härtner, Microbiol. Lett. 127, 255, (1995).

20. B. Büdel, C. Scheidegger, in Lichen Biology, Th.H. Nash III ed. Cambridge University Press, Cambridge, 1996; pp. 37-64. 